\title{
RELATION ENTRE LES EMISSIONS DE RAYONS COSMIQUES SOLAIRES ET CERTAINS SURSAUTS RADIOELECTRIQUES
}

\author{
J. F. DENISSE \\ Observatoire de Meudon, Meudon (Seine-et-Oise), France
}

Le 20 Mars 1958, L. Peterson et J. R. Winckler [1] ont observé une brève augmentation du rayonnement cosmique d'une dizaine de secondes de durée; au même instant une éruption chromosphérique se développait sur le soleil et un sursaut radio assez exceptionnel était enregistré sur ondes centimétriques et décimétriques.

D'après leurs observations, ces auteurs estiment que les rayons cosmiques étaient des rayons $\gamma$ de $0.5 \mathrm{MeV}$; c'est la première fois qu'un tel phénomène fut enregistré bien que des expériences similaires aient été effectuées en même temps que d'autres éruptions. Etant donné l'importance de cette observation qui témoigne de la formation au moment même de l'éruption de rayonnement de haute énergie, il est intéressant de décrire plusieurs caractères qui distinguent également le sursaut radio de ceux qui sont enregistrés ordinairement.

(a) Le sursaut est extrêmement bref: l'intensité augmente brusquement à $13^{\mathrm{h}} 04^{\mathrm{m}} 27^{\mathrm{s}} \pm 3^{\mathrm{s}}$, atteignant son maximum en un temps inférieur à la résolution des enregistrements ( $\sim 1$ seconde), puis décrốt exponentiellement avec une constante de temps de l'ordre de 20 secondes, une légère augmentation du niveau précède et suit l'évènement principal pendant quelques minutes. La phase intense du phénomène coincide exactement avec l'émission des rayons $r$ observée au cours d'un intervalle de temps de l'ordre de 20 secondes et qui débute vers $13^{\mathrm{n}} 04^{\mathrm{m}} 20^{\mathrm{s}}$.

(b) Son spectre est anormal: c'est, sur $3 \mathrm{~cm}$ de longueur d'onde, l'un des sursauts les plus intenses que nous ayons observés $\left(8 \times 10^{-20}\right.$ watts $\left.\mathrm{m}^{-2}(\mathrm{c} / \mathrm{s})^{-1}\right)$ et les sursauts d'une telle intensité durent toujours beaucoup plus longtemps. Sur $\lambda=21 \mathrm{~cm}$, il est 25 fois moins intense et n'est plus observable sur $\lambda=$ $60 \mathrm{~cm}$ et sur longueurs d'ondes supérieures (observations de Humain, Néra, Nançay).

(c) Le diamètre de la source d'émission est exceptionellement large: sur $\lambda=3 \mathrm{~cm}$ et $\lambda=21 \mathrm{~cm}$ le sursaut a été observé à Nançay avec des interféromètres à deux antennes espacées de $1860 \lambda$ et $500 \lambda$ respectivement, qui permettent une estimation du diamètre de la source émissive dont la dimension a été trouvée supérieure à 4 minutes d'arc sur $3 \mathrm{~cm}$ et à 8 minutes d'arc sur $21 \mathrm{~cm}$; des diamètres aussi larges ne sont observés qu'en de très rares occasions ( $M$. R. Kundu, paper 43).

A $13^{\mathrm{h}} 04^{\mathrm{m}}$ et $13^{\mathrm{h}} 05^{\mathrm{m}}$ des photographies du soleil (Fig. 1) ont été obtenues par 237 
le Service Solaire de Meudon avec l'héliographe monochromatique. Elles montrent qu'une éruption d'importance 2, brillante et localisée, s'est développée dans l'intervalle, c'est à dire au moment du sursaut, à côté d'une autre éruption qui était déjà en cours.

La parfaite concordance qui existe entre les émissions $r$ et radio suggèrent que les origines des deux rayonnements sont étroitement liées et par suite que le rayonnement radio est sans doute celui d'électrons (ou de positrons) d'énergies comparables à celle des rayons $\gamma$ : il s'agit vraisemblablement d'une émission synchrotron dans le champ magnétique du groupe de taches qui bordent l'éruption (voir aussi Takakura, paper 101).

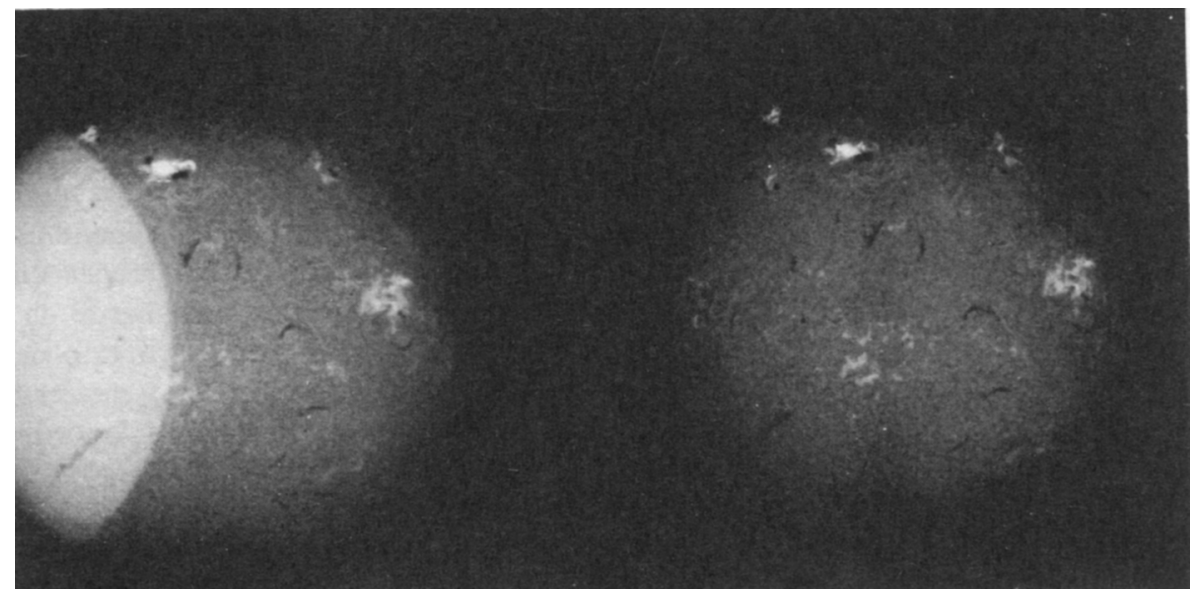

Fig. 1. Photohéliogrammes du soleil obtenus vers $13^{\mathrm{h}} 04^{\mathrm{m}}$ (d droite) et vers $13^{\mathrm{h}} 05^{\mathrm{m}}(\dot{a}$ gauche) le 20 Mars 1958; noter le développement du filanient brillant à droite de l'éruption déjà en cours sur la première photographie. (Photos aimablement communiquées par Mme M. d'Azambuja.)

L'association du sursaut centimétrique avec un rayonnement ou des particules de haute énergie est encore suggérée par le fait que la source d'émission sur $21 \mathrm{~cm}$ de longueur d'onde atteint un diamètre au moins égal à 8 minutes d'arc, soit $350,000 \mathrm{~km}$, en un temps de l'ordre de la seconde, ce qui implique une vitesse de transport de la perturbation excitatrice comparable a la vitesse de la lumière.

Bien que le sursaut radio ait été exceptionnel, on est obligé de penser qu'un grand nombre de sursauts centimétriques sont produits dans des conditions analogues à celles du 20 Mars, et que leur apparition révèle la formation, pendant une phase relativement courte de l'eruption (une fraction de minute), de particules de haute énergie.

A côté de cette émission de rayons $r$ d'énergie de l'ordre du $\mathrm{MeV}$, on sait que le soleil, en de très rares occasions, a produit des rayons cosmiques d'énergie de l'ordre du $\mathrm{BeV}$.

Unsöld [2] avait déjà noté que ces rayons cosmiques se produisaient 
en même temps que certaines émissions solaires intenses; ce type d'émission radio (type IV) a été depuis clairement identifié par Boischot [3]. En particulier pour les trois éruptions cosmiques des 25 Juillet 1946, 19 Novembre 1949, et 23 Février 1956 on peut même noter un certain parallélisme entre les courbes d'intensité du rayonnement cosmique et du rayonnement radio. D'autre part de nombreux arguments [4] indiquent que les sursauts de type IV sont sans doute dus au rayonnement synchrotron d'electrons relativistes: cette suggestion s'appuie sur le caractère très stable du rayonnement qui exclut une émission par oscillations de plasma (voir aussi J. P. Wild et al., paper 32), sur leur grande intensité qui n'est pas compatible avec une émission thermique, sur la nature du spectre qui couvre plusieurs octaves, sur la duree de vie du sursaut, de l'ordre de l'heure, qui s'explique assez bien par une disparition des électrons par collisions.

On sait que les sursauts centimétriques sont pratiquement toujours associés aux éruptions chromosphériques, pourvu que celles-ci aient une importance au moins égale à 2 , et se produisent au voisinage du début de l'éruption. Les sursauts de type IV sont au contraire beaucoup plus rares et n'accompagnent que des éruptions très importantes qu'ils suivent avec un retard qui peut atteindre 10 a 20 minutes.

En rapprochant, comme nous venons de le faire, ces deux types de sursauts des émissions de rayons cosmiques qui les ont accompagnés dans quelques cas, on est conduit à les associer à deux phases fondamentales du mécanisme de production de rayons cosmiques par le soleil.

Au moment du sursaut centimétrique s'accomplirait un processus très bref, commun à toutes les éruptions importantes, pendant lequel seraient formées dans la chromosphère des particules d'énergie moyenne de l'ordre du $\mathrm{MeV}$.

Dans une seconde phase, plus rare, prendrait place un processus de post accélération illustré par le sursaut de type IV, au cours duquel des particules seraient progressivement accélérées dans la couronne jusqu'à des énergies dont on sait qu'elles ont été dans une demi douzaine de cas supérieures au BeV.

\section{REFERENCES}

[1] Communication privée.

[2] Unsöld, A. Radio Astronomy (IAU Symposium No. 4). Cambridge, England, 1957, p. 238.

[3] Boischot, A. C.R. 244, 1326, 1957; paper 214.

[4] Boischot, A., et Denisse, J. F. C.R. 244, 2194, 1957.

\section{Discussion}

Haddock: The University of Michigan sweep-frequency receivers (100 to $580 \mathrm{Mc} / \mathrm{s}$ ) were operating during this event, but nothing was recorded because the radio intensity was too low. The $0.5 \mathrm{MeV}$ gamma rays measured by Winckler are in the mid-band of relativistic electrons required by T. Takakura to account for type IV radio bursts by synchrotron radiation. 